Volume 90 No. 4 October 2021

\section{Early Career Investigator}

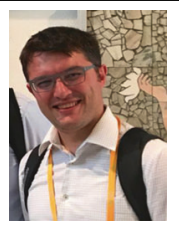

Congratulations to Erdal Sag, the Early Career Investigator, for October 2021. Dr. Sag is a pediatric rheumatologist at Ankara Training and Research Hospital in Turkey. He is from Kutahya, a small town in Turkey, and completed his medical education, pediatric residency, and pediatric rheumatology fellowship at Hacettepe University in Ankara. He completed the master's in science program of pediatric autoinflammatory diseases at the university's Institute of Child Health. Dr. Sag thanks all his mentors in rheumatology and research who have been instrumental in guiding his career, including Seza Ozen, Lucy Wedderburn, Beril Talim, and Bent Deleuran. Along with colleagues, he set up the EMERGE (EMErging RheumatoloGists and rEsearchers) group, the fellow initiative of PReS (Pediatric Rheumatology European Society). Thanks to a FOREUM International Exchange Fellowship Grant, he helped establish the Pediatric Rheumatology Unit, Translational Medicine Laboratory at Hacettepe University-the first laboratory dedicated to pediatric rheumatology research in Turkey. Dr. Sag advises researchers early in their career that "following the correct mentors, chasing every opportunity to work in different centers, collaboration with peers and seniors from all over the world, and working hard with passion will...eventually bring the success". In this issue he and colleagues report a translational research study on oligoarticular juvenile idiopathic arthritis, delineating the role of inhibitory receptors with a specific focus on LAG-3. The accompanying Comment by Mazzoni and Cimaz places this research into context. See pages 711,744 , and 720 .

\section{NEC intestinal barrier function protection by antenatal} dexamethasone and SP-D

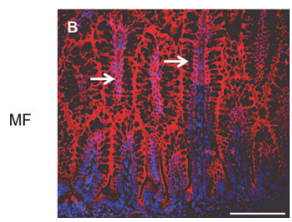

Necrotizing enterocolitis (NEC) is a devastating disorder of preterm infants. The etiopathogenesis of NEC is multifactorial, including disruption of the gut barrier function. Interventions to enhance the integrity of the gut barrier function may prevent NEC. Antenatal steroids have been shown to not only decrease the incidence and severity of respiratory distress syndrome but also decrease NEC and intracranial hemorrhage in preterm infants. Lu et al. report their investigation on surfactant protein D (SP-D) expression in the intestinal mucosal barrier after antenatal dexamethasone. They observed that antenatal dexamethasone reduced systemic inflammation, preserved intestinal barrier integrity, and stimulated SP-D expression on the intestinal mucosal surface in a rat model of NEC. In IEC- 6 cell cultures in vitro, pretreatment with SP-D blocked plateletactivating factor/lipopolysaccharide-induced tight junction disruption, indicating a role for SP-D in gut barrier integrity. See page 768 .

\section{Cannabinoids in plasma and breast milk}

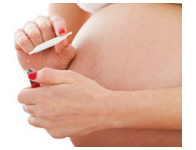

The use of cannabis among pregnant and breastfeeding women in the United States ( 5\%) has seen an increase which might be due to legalization of marijuana in some states. Nearly $70 \%$ of pregnant women consider occasional use of marijuana during pregnancy to be safe. Healthcare agencies strongly advise against cannabis use while breastfeeding. Very few studies have investigated cannabis use in lactating women or measured concentrations of cannabinoids in human milk. In a prospective study of 20 lactating mothers at a university hospital in Utah, where medical and recreational use of cannabis is legal, Moss et al. quantified the cannabinoids tetrahy drocannabinol (THC) and cannabidiol (CBD) in plasma and breast milk of breastfeeding mothers and assessed cannabis-use patterns. They found that THC and CBD accumulate in breast milk at high concentrations. They also report frequent use of cannabis by breastfeeding mothers in the early postpartum period. (Photo: ruizluquepaz/Getty.) See page 861.

\section{Skin-to-skin care and maternal singing: improved cerebral oxygenation}

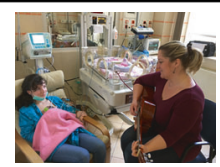

Skin-to-skin care (SSC) has been shown to have benefits for the preterm infant. Music has also been shown to have effects in infants. Meder et al. demonstrate that both SSC and maternal singing improve cerebra oxygenation. Another benefit of SSC is the impact on the infant microbiome. Casterline and Paller review the development, microbial composition, and immune correlates of the skin microbiome in early life, outlining novel therapeutic approaches targeting the skin microbiome for clinical benefit. The human microbiome plays an influential role in health and disease. Breakdown of the symbiotic relationship between microbiome and host leads to inflammation and chronic diseases of the skin, including atopic dermatitis and psoriasis, suggesting a role for microbiota in chronic diseases of other organs. The accessibility of the skin microbiota lends itself to optimization approaches that may prevent or ameliorate disease. In a related Comment, the mother of a preterm infant advocates for keeping mother and baby close together. See pages 809, 731, and 919.

\section{Advice from the SPR on sustaining} science and mentoring during COVID-19

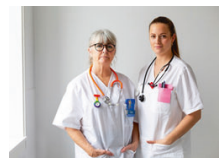

The COVID-19 pandemic has affected the professional and personal lives of nearly all of us, particularly trainees starting their professional careers, because it has significantly disrupted medical education and training. Innovative solutions are needed, including a pivot to online learning. Forster et al., for the National Pediatric Physician-Scientist Collaborative Workgroup note that "trainees are now facing unprecedented COVID-19-related disruptions in medical education, patient care experiences, research, financial stability of academic medical centers, and uncertainties about next stages of training and career opportunities, in addition to compounding challenges due to systemic racism". The group identified the concerns of trainees and propose solutions to mitigate those concerns, which is the need of the hour if we aspire to have productive physician-scientists for the future. (Photo: Johner Images/Getty.) See page 738.

\section{Residential dust lead levels and risk of lead poisoning in US children}

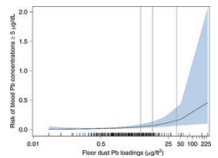

Childhood lead poisoning is a result of environmental exposure to lead at home, especially due to deteriorating lead paint, tap water from lead pipes, and, less commonly, from old toys and furniture. Blood lead concentrations, even those below $5 \mu \mathrm{g} / \mathrm{dL}$, are associated with cognitive impairments and behavioral disorders in childhood, in addition to criminal arrests, psychopathology, and lower earnings in adulthood. In 2019 the US Environmental Protection Agency (EPA) lowered the health-based standards for residential dust lead (which define the levels of lead on residential floors and windowsills that are hazardous to children) while maintaining the clearance standards for floors and windowsills that must be attained after abatement of residential lead hazards. Braun et al. investigated whether leaving the higher clearance standards in place would lead to higher blood lead levels in young children with environmental lead exposure. They report that the EPA's residential dust lead regulations place children at increased risk of lead poisoning and recommend more protective standards. See page 896 .

\section{The clinical needs of children with developmental coordination disorder}

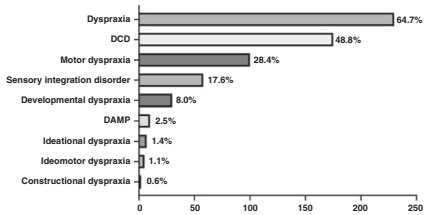

Developmental coordination disorder (DCD) is a neurodevelopmental disorder with predominant motor impairment and affects 1 in 20 school-aged children. DCD has a substantial impact on the physical social, and emotional health of children but is underdiagnosed and undersupported. One reason for the underdiagnosis may be the systemic lack of awareness of and support for DCD. Also, the clinical pathway is difficult to navigate for caregivers of children with $D C D$ who may mislabel the problem. Advocacy efforts focus on the lack of knowledge and awareness by professionals, but very few studies have examined the difficulties families have in obtaining a diagnosis and therapy for their child with DCD. Licari et al. report a survey that examined the experiences and challenges of these families. The results led to the recommendation that diagnostic guidelines be more extensively implemented into clinical practice. See page 826. 